\title{
Seismic Data Collection with Shakebox and Analysis Using MapReduce
}

\author{
Bin Tang, Jianchao Han, Mohsen Beheshti, Garrett Poppe, Liv Nguekap, Rashid Siddiqui \\ Department of Computer Science, California State Univeristy Dominguez Hills, Carson, CA 90747, USA \\ Email: btang@csudh.edu, jhan@csudh.edu, mbeheshti@csudh.edu
}

Received March 2015

\begin{abstract}
In this paper we study a seismic sensing platform using Shakebox, a low-noise and low-power 24bit wireless accelerometer sensor. The advances of wireless sensor offer the potential to monitor earthquake in California at unprecedented spatial and temporal scales. We are exploring the possibility of incorporating Shakebox into California Seismic Network (CSN), a new earthquake monitoring system based on a dense array of low-cost acceleration seismic sensors. Compared to the Phidget/Sheevaplug sensors currently used in CSN, the Shakebox sensors have several advantages. However, Shakebox sensor collects $4 \mathrm{~K}$ Bytes of seismic data per second, giving around 0.4G Bytes of data in a single day. Therefore how to process such large amount of seismic data becomes a new challenge. We adopt Hadoop/MapReduce, a popular software framework for processing vast amounts of data in-parallel on large clusters of commodity hardware. In this research, the test bed-generated seismic data generation will be reported, the map and reduce function design will be presented, the application of MapReduce on the testbed-generated data will be illustrated, and the result will be analyzed.
\end{abstract}

\section{Keywords}

Seismic Data, Shakebox, Big Data, Hadoop MapReduce

\section{Introduction}

Sitting on the tectonic boundary between the Pacific and the North American Plate, California is the state with second-most earthquakes in the United States. Large earthquakes are inevitable in California -according to the 2007 Working Group on California Earthquake Probabilities (WGCEP 2007), the probability of a magnitude 6.7 or larger earthquake striking the greater Los Angeles area before 2038 is 67\%. The recent 6.0-magnitude South Napa Earthquake, the strongest earthquake in 20 years, caused over $\$ 400$ million in damage and served as a wakeup call of how serious the situation could be.

Currently California Integrated Seismic Network (CISN, http://www.cisn.org/) is the statewide system in California for earthquake monitoring, emergency response, and loss mitigation. It has deployed around 3000 seismic stations in California and western Nevada. A seismic station includes a sensor (short-period, broadband, and strong-motion) to record the ground motion, a computer to save the data, a GPS for accurate timing and location, telemetry or radio equipment to send the data back to a processing center and a power source to run the station. 
While these stations provide high fidelity and reliable data themselves, California still lags in the number of stations needed to provide high quality of earthquake information throughout the state. Besides, being bulky and power-hungry, these stations have been costly to install and maintain, preventing them from large-scale deployment. As a result, these sparsely distributed stations only provide limited coverage and coarse-grain monitoring for earthquake.

The California Seismic Network (CSN, www.csn.caltech.edu) is a transformative approach to earthquake detection, science, and outreach. The CSN is a new earthquake monitoring system based on a dense array of low-cost acceleration seismic sensors. By producing block-by-block measurements of strong shaking during an earthquake, which are called shake maps, it can help first responders, fire fighters, rescue workers to pin down accurately the damage area in block by block level. The technical idea of CSN is that a small seismometer is hosted in each residential home or office, such that when earthquake takes place, the very granular measurement can be immediately transmitted to a centralized server (a Google cloud server) via Internet for real-time analysis. Preliminary results have shown that this system is very effective to measure earthquake damage and alert the public [1]-[4].

This paper explores four aspects that further contribute to CSN:

1) The functioning of CSN assumes the existence of the communication infrastructure. However, when the Internet is torn down during earthquake, the seismic sensors are disconnected from Google cloud via the Internet. How to preserve the large amount of data it generates during earthquake becomes a new challenge.

2) Even if Internet connection still exists, the constant transmission of the readings of hundreds of seismic sensor back to the Google cloud will inevitably overburden the communication infrastructure. Therefore, the inner data processing among sensor nodes wirelessly become more relevant.

3) The current seismic sensor node does not have wireless communication capabilities. Each seismic sensor used in CSN is operated by a small Ubuntu-based computer called SheevaPlug [7]. There is no well-defined solution to equip the current version of the SheevaPlug with wireless capability.

4) Finally, the SheevaPlug is a relatively lower-end seismic sensor, with very coarse sampling resolution.

Considering all above, we are exploring the possibility to integrate a high-precision, GPS-based, wireless, large-storage seismic sensor, called Shakebox, into CSN. In contrast to low-cost sensors used in CSN, the Shakeboxes are equipped with the most accurate strong-motion accelerometer defined by the ANSS standards. Therefore, Shakeboxes can possibly produce more precise measurements than the low-cost sensors of CSN. However, around 0.4 GB of data is generated by a Shakebox in a single day. How to process such large amount of seismic data becomes a new challenge.

In the past decade, the MapReduce programming model [10] has emerged as a popular framework for large data set analysis. The key idea of MapReduce is to divide the data into chunks, which are processed in parallel. Several open source MapReduce frameworks have been developed in the last years. In particular, Hadoop [11], the most prevalent implementation of MapReduce, has been extensively used by companies and research communities on a very large scale. In this paper, we adopt Hadoop and MapReduce for the data process and show and analyze our experimental results. Specifically, we design Map and Reduce functions that suit for the application of seismic big data.

The rest of the paper is organized as follows. Section 2 discusses the related work. In Section 3, we introduce Shakebox seismic sensing platform. Section 4 presents MapReduce and Hadoop Distributed File System. We also design MapReduce functions for Shakebox seismic data analysis. Our experiment on data collection and data analysis are illustrated in Section 5. Section 6 concludes our paper and discusses some future work.

\section{Related Work}

With the development of the micro-electro-mechanical systems (MEMS) low-cost accelerometers and the proliferation of mobile devices such as laptops and smartphones, several seismic monitoring networks that utilize low-cost and USB-based accelerometers are designed and deployed. We are aware of two projects in this line: The Community Seismic Network (CSN) [1]-[4] and the Quake-Catcher Network (QCN) [5] [6].

The CSN envisions city-wide networks of community-owned sensor devices that perform large-scale seismic sensing. It is collaboration among geophysicists, civil engineers and computer scientists to develop the sensor technologies, scalable infrastructure, and algorithmic tools needed to reliably perform large-scale seismic sensing. In contrast to traditional seismic networks that contain a small number of highly accurate sensors, the CSN 
project focuses on large numbers of inexpensive, community-held sensors, such as those in personally owned devices like smart phones.

The QCN is a distributed computing seismic network that links internal (no cost, built-in) or external (lowcost, USB-based) accelerometers connected to any participating computer for earthquake research. It is based on a distributed computing platform called Berkeley Open Infrastructure for Network Computing (BOINC) [15]. The objective of QCN is to dramatically increase the number of seismic observations by exploiting recent advances in sensing technologies and cyber infrastructure capabilities for automated warning and alert for natural disasters.

Shakeboxes have been used in ShakeNet [8], a portable wireless sensor network for instrumenting large civil structures such as buildings and bridges. The ShakeNet software subsystem is built upon Tenet [14], a programmable wireless sensing software architecture designed for tiered sensor networks.

In contrast to all above Shakebox-related research, we are approaching the Shakeboxes from the perspective of big-data processing, and design Map and Reduce functions to process the seismic data generated by Shakeboxes.

There are a few researches in recent years that focus on big sensor data. Lee et al. [9] designed a platform that enables sensor data to be taken from collection, through use in models to produce useful data products. They propose a response through a sensor data platform "Concinnity", which can take sensor data from collection to final product via a data repository and workflow system. They summarize the key features of their approach and explore how it enables value to be derived from sensor data efficiently. Liu et al. [12] propose an integrated method to address the heterogeneity issue in modeling big time series sensor data. They present both linear and nonlinear feature extraction techniques, as well as a procedure to determine the right extraction method for individual time series. Guo et al. [13] observe that model-based sensor data approximation reduces the amount of data for query processing. They propose an innovative index for modeled segments in key-value stores, called KVI-index. They show their approach outperforms in query response time and index updating efficiency both Hadoop-based parallel processing of the raw sensor data and multiple alternative indexing approaches of modelview data.

In contrast to above big sensor data researches, most of which focus on mathematical modeling of big sensor data, we are particularly interested in the whole process of real seismic sensor data collection, analysis, and visualization.

\section{Shakebox Seismic Sensing Platform}

Shakexbox is a wireless sensor node equipped with a low-noise and low-power 24-bit triaxial accelerometer. The use of low-noise and low-power 24-bit accelerometer puts ShakeBox near the US Geological Survey's "Class A" device specifications for earthquake measuring instruments. The system comes preloaded with sensing software as well as deployment tools that enable rapid deployment. Small form factor and portable design makes Shakeboxes easy and fast to deploy. Shakeboxes have been used in structural health applications [8], since aforesaid small form factor and portable design help in capturing the structural health of the building, bridge, dam or tunnel expeditiously when compared to wired monitoring methods. Shakeboxes have their own power source, making them independent of the infrastructure. This is vital for remote locations or in disaster struck areas.

The Shakeboxes were manufactured by Refraction Technologies (RefTek, http://www.reftek.com/). Figure 1 is a modular design paradigm for the ShakeBox. Figure 1(a) shows several visible modules: Power, GPS, Radio, and Communication. Figure 1(b) shows the CPU, Power and A/D modules inside the Shakebox. These modules are housed in a custom-made weatherproof casing.

The CPU module contains the system processor board (a Crossbow iMote2 mote) and the RT617 CPU carrier board and controls all system operations. The boards are housed in an electro magnetic shield to reduce external effects on the analog module.

The iMote2 mote controls the communication to other three modules. IMote2 is an advanced sensor network platform and consists of a Marvel PXA271A ARM CPU, which is a 32 bit microcontroller, and a CC2420 radio, an 802.15.4 compliant 2.4 GHz wireless communication radio with up to $256 \mathrm{Kbps}$ bit data rate. Dynamic scaling of core frequency of the PAX271 microcontroller from $13 \mathrm{MHz}$ to $208 \mathrm{MHz}$ provides a varied range of options for balancing processing power with energy usage.

The Power module provides the power requirements of the different components and consists of RT618 FPGA board and RT620 power board. RT618 provides communication with CPU module, a clock, control of 


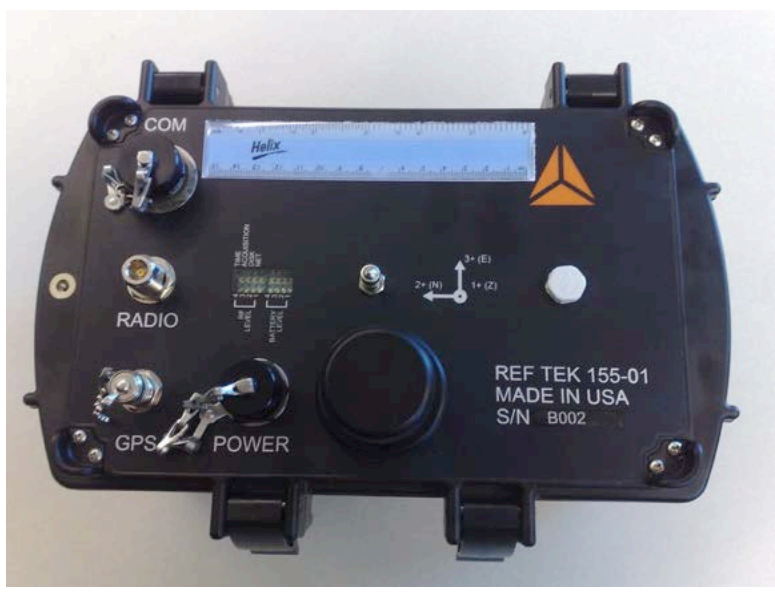

(a)

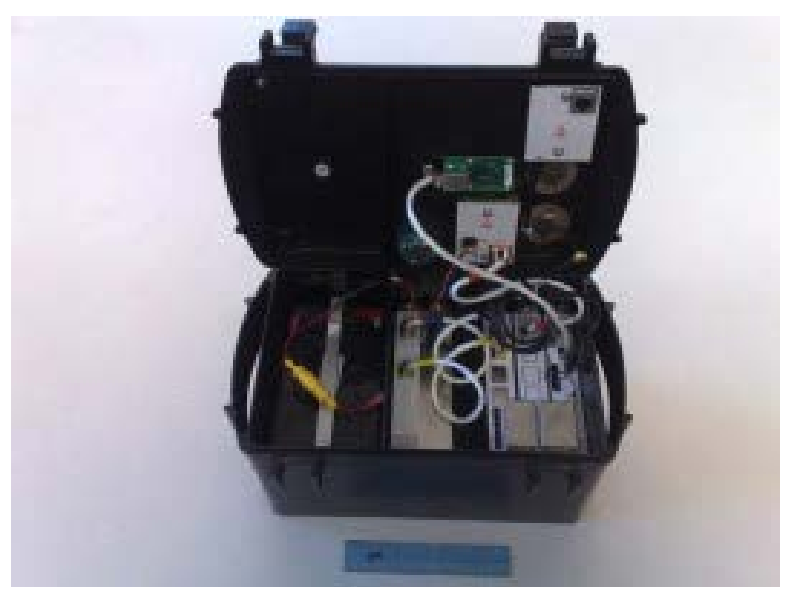

(b)

Figure 1. a) Shakebox with weatherproof enclosure with 6-inch ruler shown for scale; b) Placement of detailed modules with 6-inch ruler shown for scale. Source: http://nsl.cs.usc.edu/Projects/ShakeNet

the voltage monitor A/D converter, control of analog power supplies and board ID EEProms. RT620 provides an input power controller, switching supplies at different voltage levels, a 16-bit A/D monitor for supply voltages and input currents, and a board ID EEProms.

The sensor module consists of three Colibyrs SiFlex 1500 accelerometers, which are interfaced to the RT614 board in the A/D module. The SiFlex1500 operates from a bipolar power supply voltage that can range from \pm 6 $\mathrm{V}$ to $\pm 15 \mathrm{~V}$ with a typical current consumption of $12 \mathrm{~mA}$ at $\pm 6 \mathrm{~V}$. The linear full acceleration range is $\pm 3 \mathrm{~g}$ with a corresponding sensitivity of $1.2 \mathrm{~V} / \mathrm{g}$. The sampling rate of the sensor module is $10,100,200$, or 1000 samples per second.

The weatherproof casing houses all the modules. Each module is electronically shielded to protect against electromagnetic disturbance. The lead acid battery used in the Shakebox is placed in a separate sealed compartment to isolate it from the electronics in case of battery leakage. The box provides serial connectors, connector for GPS, LEDs for display and feedback and antenna connector for high gain external antenna used by iMote2's radio. It has three screws and a spirit level for leveling. The prototype box in Fig. 1 is made up resin plastic but the production pieces will be metallic aluminum.

Finally, the Shakebox is equipped with one or two Compact Flash Type I or Type II storage media (disks). CF flash storage is available up to 16 GB capacity. For example, 4 GB is enough storage to hold more than 100 days of three channel, 100 sample per second data recorded with compression. Files are written in FAT32 format allowing high capacity disks to be used.

\section{MapReduce Functions for Shakebox Data Analysis}

MapReduce is a distributed processing framework that enables big data processing. The Hadoop distributed file system (HDFS) is a distributed, scalable, and portable file-system written in Java for the Hadoop framework. Below we present both and show how we adopt them in our seismic data analysis.

MapReduce [10] has two main components, a mapper and a reducer. A mapper works on each individual input record to generate intermediate results, which are grouped together based on some key and passed on to the reducers. A reducer works on the group of intermediate results associated with the same key and generates the final result using a result aggregation function. The processing units of the MapReduce framework are key-value pairs.

Hadoop Distributed File System (HDFS) is a Java-based file system that provides scalable and reliable data storage that is designed to span large clusters of commodity servers. HDFS splits files into large blocks (default $64 \mathrm{MB}$ or $128 \mathrm{MB}$ ) and distributes the blocks amongst the nodes in the cluster.

Hadoop MapReduce framework will create one map task for every input task specified by the input format for the job. A job is a method in which MapReduce assigns a task to be processed in a manner specified by the user created configuration to evaluate key/value pairs from input to intermediate records. 
The earthquake data is produced through experiment with the Shakebox. REF TEK 130S is used as input for the MapReduce program. The data in the file is formatted into four tab-delimited columns, each containing data of the type "double”.

Figure 2 and Figure 3 show the Map and Reduce functions that we designed, respectively. When the program is launched, the map function reads the input file line by line. It then maps each data item from the line based on the algorithm provided. In our case, the algorithm takes, for each column, the first value and subtracts it from the next. The absolute value of the result is saved to an array of doubles. As the array is progressively filled up, the data contained in the array is summed and then divided by the number of elements in the array to find the average value.

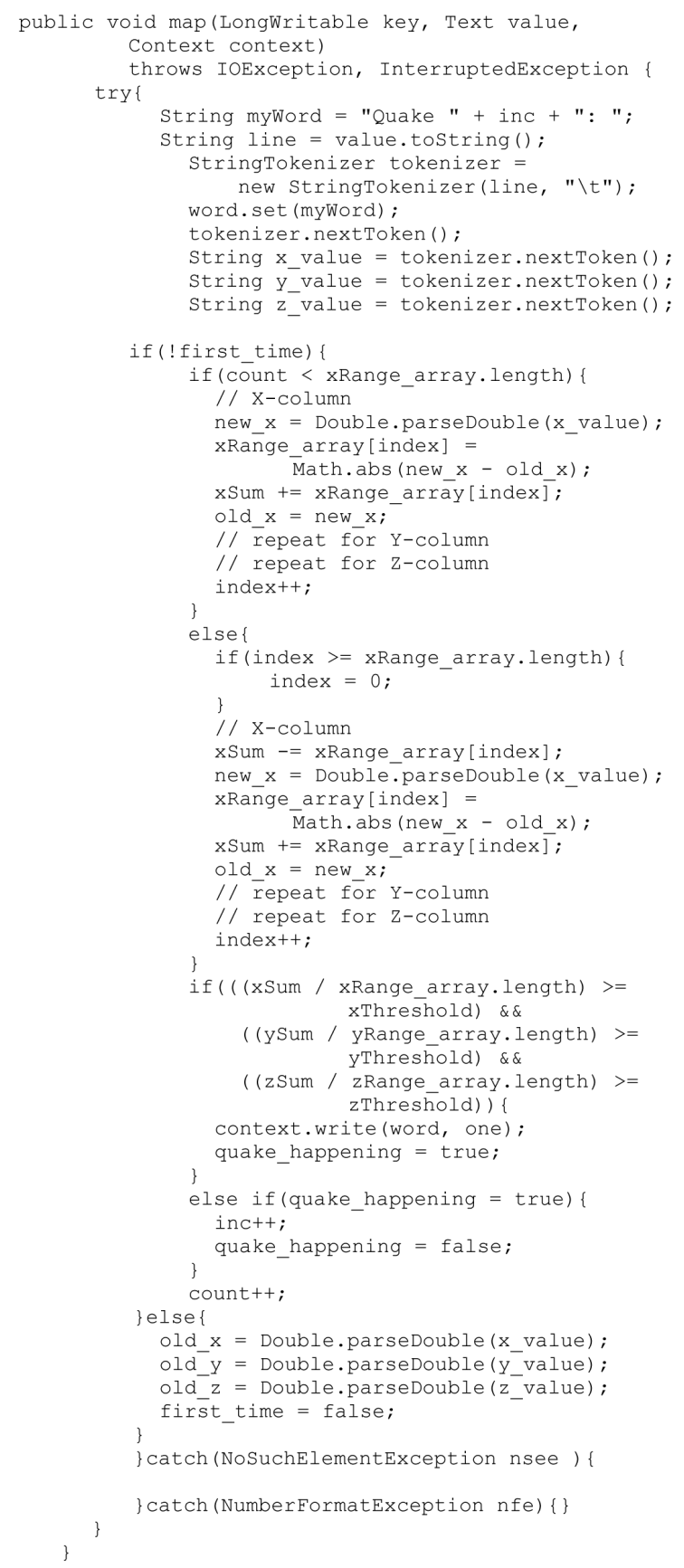

Figure 2. Map function. 


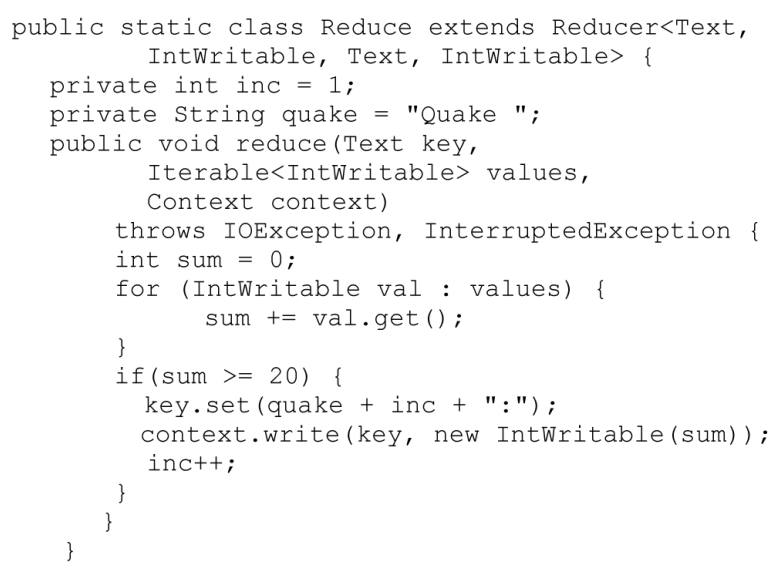

Figure 3. Reduce function

If the result of this operation is less than a threshold value we hardcoded, then the program understands that nothing is happening. If the result of this operation is greater than the threshold value, then the program understands that an event is happening. The event in this case is the first wave of an earthquake. Therefore, the program generates an output pair such as ("Quake 1:", 1) to indicate that 1 tremor has been recorded for the first wave of the earthquake. If a second tremor occurs for the first wave, then another key/value pair ("Quake 1:”, 1) is recorded. And so on.

The array represents a range over which the average of the differences between consecutive values has to be greater than the threshold value in order for us to consider that an earthquake is happening. We chose a threshold of 0.5 and an array of 100 elements for our range. When the end of the array is reached, the oldest value, which is located at index 0 , is removed, and the next value from the input file is loaded at that index. Therefore, as the mapping programs goes through the file, we always have 100 values that are being considered to determine whether or not an earthquake is happening.

If the average of the difference of those 100 elements falls below the threshold value after being higher than that for a while, then we consider that the first wave of an earthquake that was happening has stopped. At this moment, we start looking for the next wave. Repeat this process until we reach the end of the file.

At this point, the data mapped into the context of the MapReduce program is shuffled and sorted. During this process, all the data belonging to "Quake 1", which indicates the first wave of the earthquake, is put into a key/ value pair. The word "Quake 1" is used as the key, and every other item that was coupled with the key "Quake 1:" in the output of the map function is put into a list. That list is identified as the value. The same process is repeated for "Quake 2:”, “Quake 3:”, and so on, if they exist.

Then, each key/value pair is sent to the reduce function. This function identifies each key and iterates over the list of value to sum them up. The total for each key is saved to the output file. And by repeating this process for each key and its associated values, we obtain a file that shows how many waves of earthquake occurred, and for each wave the total number of tremors.

\section{Experiment}

We have adopted 4 machines, each machine has Xeon processors, 2 TB disc storage, and 12 GB RAM. As raw data is received from the sensor nodes, it is saved to the HDFS. The data can be stored in the temp directory until processed. A job is created to process the data using the MapReduce functions. The output data is saved in a directory intended to be the input of a java visualization chart program. The program uses the data set start and finish points to output a graph depicting the changes in movement for the $\mathrm{X}, \mathrm{Y}$, and $\mathrm{Z}$ planes. The graphs can be saved for future analysis or analyzed in real time. The final graph file system will not include idle time data, which in turn will reduce the state space and search space for future analysis. Future programs to implement in the MapReduce environment can include mapping the comparison of the three graphs and reducing the data to anomalous events for future research.

The initial mapping tasks are created as jobs and assigned to individual nodes for processing. The NameNode 
keeps track of each job and follows the user configuration of the mapping function to assign file location and control settings.

After all mapping tasks have been executed as jobs, the status of those results is displayed. It also specifies some configuration settings as well as some logged statistics including resource calculations.

As jobs are completed from the mapping functions, the reduce functions process the intermediate values and the meta-data associated with the completed tasks are processed by the NameNode. The NameNode keeps track of how much data is written and where it is located and what the data represents. During this process all completed jobs are logged for analysis.

The output data from the MapReduce process contains specific data sets that were sought from our MapReduce program. The output is specific sections of the input data that meet our minimum requirements for identifying an earthquake. Data that was recorded when no earthquakes were occurring is omitted from the results. This reduces the amount of data that needs to be stored.

Finally, Figure 4 shows a visualization of a specific seismic data set from one Shakebox output, which is processed by our MapReduce functions on the larger data set. Figures 4(a)-(c) show the Shakebox movement along $\mathrm{X}$-, $\mathrm{Y}$-, and $\mathrm{Z}$ - axis respectively. The $\mathrm{X}$ plane graph is the movement along the $\mathrm{X}$-axis of the shakebox (if you view it from above); the $\mathrm{Y}$ plane graph represents the movement along the $\mathrm{Y}$-axis as you look at the box from above; the $\mathrm{Z}$ plane graph represents the movement of the shake box when it is lifted up and down.

\section{Conclusion and Future Work}

In this paper we studied a seismic sensing platform using Shakebox, a low-noise and low-power 24-bit wireless

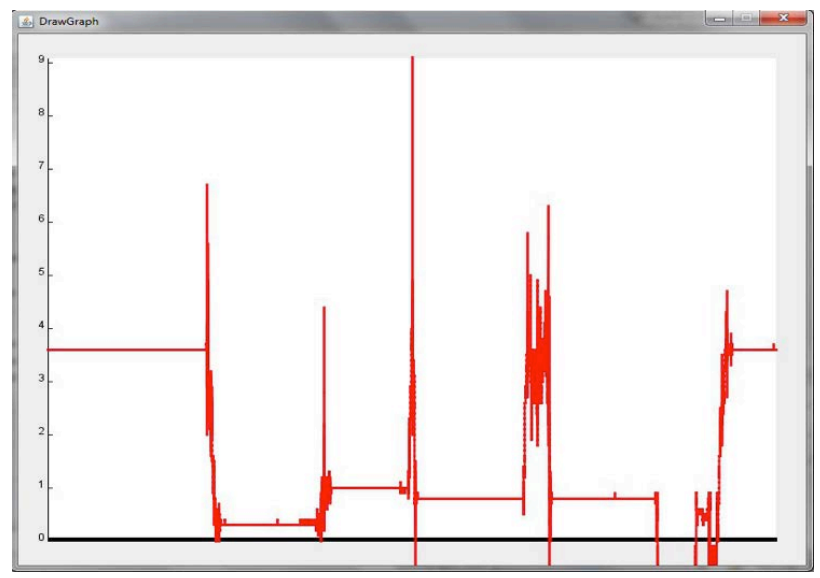

(a)

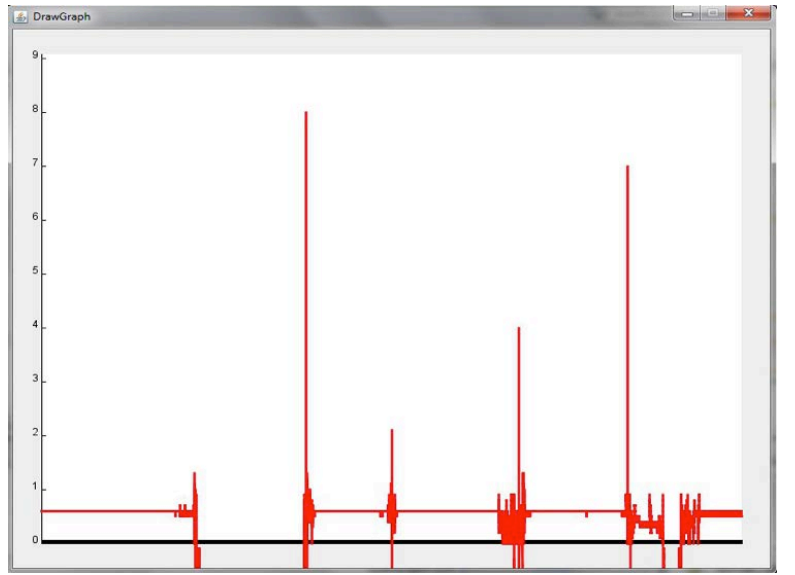

(a)

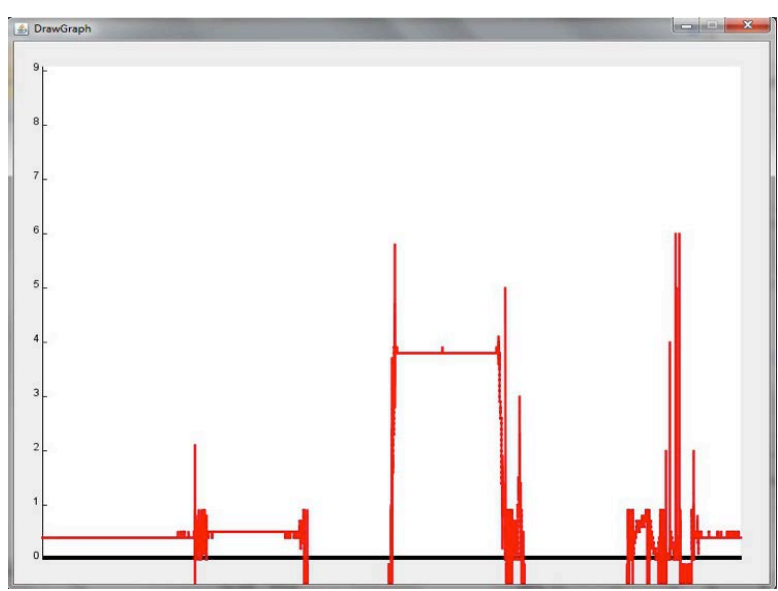

(b)

Figure 4. Shakebox movement. (a) X Plane; (b) Y Plane; (c) Z Plane. 
accele rometer sensor. To process large amount of seismic data from this platform, we adopted Hadoop/ MapReduce. We designed map and reduce functions on the testbed data and analyzed the result. As ongoing effort and future work, we are working on the following two directions. Currently, the Shakeboxes are invoked from command line and only collect data for a specified amount of time. We are configuring Shakeboxes such that it can constantly collect data. This is critical to catch real earthquake occurrences. Second, we are planning to incorporate real earthquake models into our Haddop/Mapreduce analysis, to better evaluate its efficacy.

\section{Acknowledgements}

We would like to thank Dr. Monica Kohler for providing the Shakeboxes and many helpful suggestions and the Caltech Community Seismic Network team for many opportunities of participation and discussion. This work was supported in part by the NSF Grant CNS-1116849, and in part by RSCA program of CSUDH.

\section{References}

[1] Faulkner, M., Clayton, R., Heaton, T., Mani Chandy, K., Kohler, M., Bunn, J., Guy, R., Liu, A., Olson, M., Cheng, M. and Krause, A. (2014) Community Sense and Response Systems: Your Phone as Quake Detector. Communications of the ACM, 57, 66-75. http://dx.doi.org/10.1145/2622633

[2] Clayton, R.W., Heaton, T., Chandy, M., Krause, A., Kohler, M., Bunn, J., Guy, R., Olson, M., Faulkner, M., Cheng, M., Strand, L., Chandy, R., Obenshain, D., Liu, A. and Aivazis, M. (2012) Community Seismic Network. Annals of Geophysics, 54.

[3] Olson, M., Liu, A., Chandy, K.M. and Faulkner, M. (2011) Rapid Detection of Rare Geospatial Events. 5th ACM International Conference on Distributed Event-Based System.

[4] Faulkner, M., Olson, M., Chandy, R., Krause, J. and Chandy, K.M. (2011) The Next Big One: Detecting Earthquakes and Other Rare Events from Community-Based Sensors. 10th International Conference on Information Processing in Sensor Networks (IPSN).

[5] Cochran, E.S., Lawrence, J.F., Kaiser, A., Fry, B., Chung, A. and Christensen, C. (2011) Comparison between Low-Cost and Traditional MEMS Accelerometers: A Case Study from the M7.1 Darfield, New Zealand, Aftershock Deployment. Annals of Geophysics, 54, 728-737.

[6] Cochran, E., Lawrence, J., Christensen, C. and Chung, A. (2009) A Novel Strong-Motion Seismic Network for Community Participation in Earthquake Monitoring. IEEE Inst \& Meas, 12, 8-15.

[7] https://www.globalscaletechnologies.com/t-sheevaplugs.aspx

[8] Mishra, N., Hao, S., Kohler, M., Govindan, R. and Nigbor, R. (2010) ShakeNet: A Tiered Wireless Accelerometer Network for Rapid Deployment in Civil Structures. http://nsl.cs.usc.edu/Papers/?action=download\&upname=Mishra10a.pdf

[9] Lee, C.-H., Birch, D., Wu, C., Silva, D., Tsinalis, O., Li, Y., Yan, S.L., Ghanem, M. and Guo, Y.K. (2013) Building a Generic Platform for Big Sensor Data Application. IEEE International Conference on Big Data. http://dx.doi.org/10.1109/BigData.2013.6691559

[10] Dean, J. and Ghemawat, S. (2004) Mapreduce: Simplified Data Processing on Large Clusters. 6th Conference on Symposium on Opearting Systems Design \& Implementation, 137-150.

[11] The Apache Hadoop Framework. 2013. http://hadoop.apache.org

[12] Liu, B., Chen, H.F., Sharma, A., Jiang, G.F. and Xiong, H. (2013) Modeling Heterogeneous Time Series Dynamics to Profile Big Sensor Data in Complex Physical Systems. IEEE International Conference on Big Data.

[13] Guo, T., Papaioannou, T.G. and Aberer, K. (2013) Model-View Sensor Data Management in the Cloud. IEEE International Conference on Big Data. http://dx.doi.org/10.1109/BigData.2013.6691585

[14] Paek, J., Greenstein, B., Gnawali, O., Jang, K.-Y., Joki, A., Vieira, M., Hicks, J., Estrin, D., Govindan, R. and Kohler, E. (2010) The Tenet Architecture for Tiered Sensor Networks. ACM Transactions on Sensor Networks (TOSN), 6(4). http://dx.doi.org/10.1145/1777406.1777413

[15] http://boinc.berkeley.edu 